\title{
CLOCHES, SONS ET CLOCHERS. SENS VISUELS ET ACOUSTIQUES AU MOYEN ÂGE
}

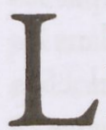

es grands clochers du Moyen Âge remplissaient une multitude de fonctions: échauguette pour les gardiens de la ville soucieux de détecter tout type de dangers, comme les ennemis ou les incendies; tribune pour les musiciens; phare symbolique de la prospérité et de la fierté de l'institution concernée; belvédère pour les princes affirmant leur pouvoir sur les territoires qu'ils dominaient. Mais de prime abord les clochers servaient - et servent toujours - à loger les cloches, l'instrument acoustique essentiel du Moyen Âge. On sait qu'elles servaient à rythmer le temps de la vie séculière et spirituelle, de sorte que le droit de disposer de leurs sons comptait parmi les privilèges essentiels des différents pouvoirs. Ce n'est pas seulement dans un sens temporel que les sons de cloches créèrent un espace juridique - par exemple en indiquant l'ouverture et la fermeture des portes de la ville ou des comptoirs de vin -, puisque dans certains cas, cette capacité pouvait s'étendre à une délimitation topographie. Ainsi, à Metz, un document de 1319 précise la limite de la banlieue selon l'audibilité de la grande cloche de la cathédrale. Dans cette perspective, Alfred Haverkamp a souligné la spécificité des cloches dans la définition de la communauté et de la commune, telle qu'elle était conçue dans la civilisation chrétienne occidentale' Les communes se constituent en effet par les actions et les intérêts de complexes « publicités », très souvent en concurrence entre eux, et dépendant des places publiques de rassemblement ainsi que de signaux acoustiques clairs et forts. Cette fonction essentielle des cloches n'est pas sans conséquence pour l'archéologie médiévale car elle nous incite à réfléchir sur les édifices d'où émanaient les sons et sur les endroits où les cloches étaient suspendues. Leur nombre, leurs dimensions, leur poids, la technique et surtout l'altitude de leur suspension ont un rapport direct, à la fois technique et esthétique, avec les constructions susceptibles d'abriter les cloches et leurs beffrois. Cette question n'a pourtant été traitée qu'à de rares exceptions et la littérature campanologique se concentre très nettement sur

\footnotetext{
I Alfred HAVERKAMP, « “... an die große Glocke hängen”. Über Öffentlichkeit im Mittelalter », Jahrbuch des Historischen Kollegs 1995, p. 71-I12; ID., Gemeinden, Gemeinschaften und Kommunikationsformen im hohen und späten Mittelalter. Festgabe zur Vollendung des $6_{5}$. Lebensjahrs, dir. F. BURGARD, L. CLEMENS et M. Matthaeus, Trèves, Kliomedia 2002, p. 277-313; Edmund KizıK, «Die Funktion von Glockeninschriften. Ein Versuch ihrer Einteilung unter methodologischem Aspekt », Jahrbuch der Glockenkunde 13-14, 2001-2002, p. 1-12.
}

Regards croisés sur le monument médiéval, éd. par Marcel Angheben, Pierre Martin et Éric Sparhubert, Turnhout 2018 (Culture et société médiévales, 33), p. 445-456 
les instruments eux-mêmes, négligeant presque complètement les rapports entre cloches et clochers².

Pour mieux comprendre ces relations, il convient de souligner brièvement le caractère sacré des cloches. En Europe, elles renaissent à l'époque mérovingienne et dans un contexte monastique pour indiquer les heures canoniales et structurer la liturgie, et cette sacralité s'est maintenue jusqu'à nos jours puisque toute cloche religieuse est baptisée et bénie. Très souvent, les cloches appartenaient à une institution religieuse, même si leur fabrication était généralement financée par les communes désireuses d'en orner les grandes églises paroissiales, comme dans les villes impériales du Moyen Âge tardif. La dimension sacro-liturgique des cloches comprend également certaines fonctions apotropaïques: repousser les menaces diaboliques, les fléaux et les ennemis militaires. Ces aptitudes multiples sont également exprimées dans les formules de bénédiction des cloches qui correspondent à celles de bénédictions et de prières presbytérales. La cloche a donc valeur d'intermédiaire entre la commune terrestre et le pouvoir protecteur émanant de Dieu. Cela se traduit dans les sept valeurs cardinales de la cloche qui, dans certains cas, s'expriment explicitement dans les inscriptions qui y sont appliquées, comme par exemple à Haguenau, en 1268 : «Je chante la louange du vrai dieu, convoque le peuple, rassemble le clergé, lamente les morts, expulse la peste, orne les fêtes, et les démons redoutent ma voix $\gg^{3}$. Dès le $\mathrm{X}^{\mathrm{c}}$ siècle, les cloches subissent aussi une allégorèse typologique, considérées soit comme le renouvellement des trompettes d'argent de Moïse, par lesquelles celui-ci a convoqué son peuple au sacrifice, soit en tant que trompettes qui ont démantelé la ville de Jéricho ${ }^{4}$.

Quant aux inscriptions des cloches, il convient de souligner qu'elles parlent très souvent à la première personne: par leur haute voix, elles adoptent plus leur propre expression qu'elles ne délivrent un contenu spirituel - le message des saints -, exception faite des cloches dédiées à la Vierge (Ave Maria). En fait, chaque cloche est dédiée à un ou plusieurs saints patrons dont le choix

2 Art. «Glocke», Reallexikon für Antike und Christentum, II, col. 164-196; Art. «Cloche» et «Clocher », Dictionnaire d'archéologie chrétienne et de liturgie, III, 2, col. 1954-1982; Christhard Mahrenholz, Glockenkunde, Kassel-Bâle, Bärenreiter, 1948; Percival Price, Bells and Man, Oxford, Oxford University Press, 1983; Lusus campanarum. Beiträge zur Glockenkunde. Sigrid Thurm zum 80. Geburtstag, dir. T. Breuer, Munich, Landesamt für Denkmalpflege, I986 ( «Arbeitshefte des bayerischen Landesamtes für Denkmalpflege », 30); Kurt KrAMER, Die Glocke und ihr Geläute: Geschichte, Technologie und Klangbild vom Mittelalter bis zur Gegenwart [1 $\mathrm{r}^{\mathrm{e} r}$ éd. 1986], Munich; Deutscher Kunstverlag, 1990; Carl-Rainer SCHAD, Wörterbuch der Glockenkunde. Stichwörter zur Campanologie mit Erläuterungen und Literaturhinweisen, Berne-Stuttgart, Hallwag, 1996; Kurt KRAMER, Glocken in Geschichte und Gegenwart: Beiträge zur Glockenkunde, Karlsruhe, Badenia, 1997; Thierry Gonon, Les cloches en France au Moyen Age. Archéologie d'un instrument singulier, Paris, Errance, 2010.

3 A. HAVERKAMP, « “... an die große Glocke hängen”... », p. 285.

$4 \mathrm{Nb}$ IO, I sq; Jos 6, 4. Sabine Ż ${ }_{\mathrm{AK}}$, Musik als « Ehr und Zier» im mittelalterlichen Reich: Studien zur Musik im höfischen Leben, Neuss, Päffgen, 1979, p. 38. 
correspond souvent aux vocables de l'église concernée. La cloche participe donc concrètement à la personnalité de chaque saint qu'elle représentes: elle agrandit et intensifie son être et le rend accessible grâce à une large diffusion sonore. Ceci est un trait essentiel des messages des saints dont - suivant les paroles de Bernard de Clairvaux -, le sonus - il se réfere aux apôtres! - in omnem terram exivit afin que ceux-ci clamant ... in publico ... in nubes volant ${ }^{6}$. Cette spécificité des voix sacrées peut être directement attribuée aux cloches, grâce à leurs sons puissants portant très loin. Dans cette perspective, il faut aussi relever l'interprétation proposée par Guillaume Durand qui, dans son Rationale divinorum officiorum, résume ainsi les significations des cloches: elles symbolisent les voix fortes et emphatiques des prophètes et des prédicateurs ${ }^{7}$. Ce sens quasi personnel des cloches se concrétise, dès le XIV ${ }^{\mathrm{e}}$ siècle, par des représentations en bas-relief appliquées sur leur surface. Souvent, une telle pratique se combine avec la présence d'insignes de pèlerinage. Les matrices ont été pressées sur la face intérieure du moule de fonte extérieur et reproduisaient ainsi leurs formes en relief dans le corps de bronze de la cloche. La piété qu'avaient éprouvée ces pèlerins résonnait donc à chaque coup de cloche.

L'audibilité de ces voix sacrées était encore amplifiée par la durée du battement ainsi que par la résonnance, parfois considérable, de chaque cloche. Or, la puissance du son s'avérait essentielle: une multitude de documents médiévaux atteste l'importance attribuée au volume acoustique et au bruit solennel pour légitimer un pouvoir revendiqué ou pour se défendre contre des puissances hostiles. Il s'agissait là d'une tradition de superstition qui prétendait pouvoir distancer les démons et les malheurs à l'aide de grand bruit et de cris puissants ${ }^{8}$. En ce sens, le volume des sons des cloches n'a pas pour unique vocation d'émettre des signaux acoustiques à l'adresse d'une collectivité. De surcroît, le volume du son renvoie à des questions de hiérarchie spirituelle, comme l'indique explicitement l'inscription de la vieille cloche d' « Hosanna » de la cathédrale de Mayence, fondue en 1298: OSANNA HEISINICH DIE DONE ALLER MENTZER GLOCKEIN UBERDON ICH (Je m'appelle Hosanna, je couvre les sons de toutes les autres cloches mayençaises) ${ }^{9}$. Et dans certains cas, les textes attestent que la puissance des sons devait assurer une bonne communication avec le ciel. Une des cloches de

5 E. KızıK, « Die Funktion von Glockeninschriften... », ici p. 3.

6 BeRNARD DE ClairvauX, Sermones in cantica canticorum, dans Opera genuina, III, Paris, 1833, p. 363.

7 Guillaume Durand, Rationale divinorvm officiorvm, I, 4, éd. A. Davril, Turnhout, Brepols, ig95

(Corpus Christianorum. Continuatio Mediaevalis, 140/I), I, p. $52-57$ (De campanis).

8 S. Ż ${ }_{\mathrm{AK}}$, Musik als « Ehr und Zier »...

9 Konrad Bund, Claus Peter, «Die Glockengüsse des Meisters Gherardus de Wou zu Erfurt im Jahre I497 », Jahrbuch der Glockenkunde I-2, 1989-1990, p. 37-65. 
Saint-Victor de Xanten, par exemple, évoque le saint patron afin qu'il demande à Dieu de lui conférer les dons salvateurs : pete dona salutis ${ }^{10}$.

Or, ce n'est que vers la fin du XII ${ }^{c}$ et le début du XIII ${ }^{c}$ siècle, période également décisive pour l'architecture gothique, que la durée de résonnance ainsi que le volume des sons s'améliorent de façon significative. Ce changement est dû à de nouvelles formes de cloches et aux techniques de fonte. La cloche en forme de «pain de sucre » remplace désormais la cloche en forme de « ruche» pour finalement adopter, dès le XVIc siècle, la forme « gothique » encore plus évasée. Cela engendre une nouvelle qualité des sons des cloches, désormais plus différenciés et variés, ce qui permettra une gamme plus large des signaux collectifs. En même temps, il sera bientôt question d'un accordage harmonique individuel et collectif au sein du clocher: c'est ce qu'illustre le traité de musique de Jérôme de Moravie, composé au dernier quart du XIII' siècle dans un milieu dominicain, en France septentrionale. Le chapitre 18 est explicitement consacré à la question de la bonne consonance devant s'exprimer, par une relation étroite entre le poids de la cloche et sa hauteur de son ${ }^{\text {Ir }}$.

C'est avec la forme en «pain de sucre » de la cloche qu'est née sa qualité acoustique si caractéristique. Le coup du battant contre la pince (lèvre inférieure) fait vibrer la robe de la cloche qui émet une composition acoustique spécifique d'harmoniques supérieures, de durées diverses et d'intervalles caractéristiques. Le calcul de ces profils harmoniques dépend essentiellement du poids, de la taille, du métal de fonte et du profil spécifique de la cloche entre les parties du cerveau, l'épaule, la robe, la panse et la pince. Le calcul préliminaire et très précis de ces facteurs, et donc de l'accordage parfait des sonneries, se perfectionnera jusqu’à la fin du Moyen Âge. Cette harmonie musicale deviendra même une valeur artistique en soi, parfois formulée par les inscriptions des cloches. En 1497, le célèbre fondeur néerlandais Gérard de Wou fabriqua deux nouveaux instruments pour la cathédrale d'Erfurt, dont la fameuse « Gloriosa » avec ses tons très bas. Une de ces cloches louangeait, par son inscription, la qualité de modulation harmonique de l'ensemble de la nouvelle sonnerie: « Grâce à l'art de Gérard de Kampen [Gérard de Wou] on chante en l'honneur du Dieu trinitaire: Ce qui est: moi, je suis sol [quinte à la fondamentale], Gloriosa est ut [fondamentale], mais mi est Osanna [tierce majeure], donc le plenum englobe une Quinte [triple accord en mode majeur]. En l'an du Seigneur I497 ». Un accord très semblable et explicitement harmonique (ad probationem consonancie) avait été réalisé en I 490 pour la sonnerie de la cathédrale métropolitaine de Mayence ${ }^{12}$. L'adaptation de la modulation acoustique d'Erfurt n'était certainement pas conçue sans préméditation, de façon à signaler son statut de collégiale de Mayence.

Io E. KIZIK, « Die Funktion von Glockeninschriften... », p. 9.

in Jérôme de Moravie, Tractatus de musica, éd. E. de Coussmemaker, Scriptorum de musica medii aevi, n. s., I, Paris, Durand, 1864 , p. 73.

I2 K. Bund, C. Peter, « Die Glockengüsse des Meisters Gherardus... ». 
Ce calcul très précis de la sonorité permettait aussi la reconstruction des qualités musicales de cloches anciennes ou détruites, ainsi que le maintien des traditions acoustiques des villes, églises, hôtels de villes, etc. Cette maîtrise était susceptible de créer des valeurs symboliques, par exemple par un accord parfait de trois sons comme une référence musicale à la Trinité. Etant donné les compétences hautement spécialisées demandées aux fondeurs de cloches, on comprend leur statut social élevé et leur très bonne rémunération à l'époque médiévale. De plus, le bronze très coûteux manipulé par les fondeurs demandait d'énormes investissements financiers et logistiques, sans compter qu'une fonte ambitieuse risquait facilement d'échouer. Au cours des XIII ${ }^{\mathrm{e}}-\mathrm{XV}{ }^{\mathrm{e}}$ siècles, les ambitions des maîtres d'ouvrage ecclésiastiques et communaux s'accrurent et augmentèrent les défis techniques: une cloche plus lourde demandant non seulement plus de métal, mais aussi de savoir-faire et une logistique plus avancée ainsi qu'un beffroi techniquement capable de la suspendre et de résister à un balancement fréquent. Durant la même époque, on constate une tendance au gigantisme et à de véritables prouesses techniques, comme l'atteste la « Cardinal d'Amboise » de la cathédrale de Rouen qui pesait I7 tonnes ou la « Maria Regina » de 2 I tonnes fondue en isig pour la cathédrale de Strasbourg, mais brisée deux ans plus tard. Mise à part la fabrication coûteuse de ces cloches colossales, les conséquences logistiques furent considérables : leur dimension ne correspondait pas toujours à l'espace disponible dans les chambres des cloches. Ainsi, à la cathédrale de Bamberg, pour permettre un libre balancement de la « Heinrichsglocke » (cloche Henri II) fondue en I3II, il fallut enlever une partie de la maçonnerie à l'intérieur de la tour nord-est ${ }^{13}$. Faire sonner ces grandes cloches demandait aussi de grandes équipes de sonneurs, s'élevant jusqu'au nombre de douze personnes... qu'il fallait bien entendu payer.

Tout ceci fait comprendre l'importance et l'ambition qu'il convient d'attribuer à la construction des clochers. Abriter les cloches ne se résume pas à mettre à disposition un étage en hauteur. Une construction stable et résistante aux vibrations du balancement devait se conjuguer avec les effets acoustiques désirés, surtout en ce qui concerne la direction et le rayon de diffusion du son. La chambre des cloches forme, avec sa voûte, un espace de résonnance qui transforme et dirige les sons des instruments. De plus, la mise en œuvre d'un clocher ne s'explique pas uniquement par la volonté de disposer d'un lieu pratique pour abriter les cloches; elle vise également à rendre visible, littéralement et symboliquement, les voix puissantes des saints. Malheureusement, on ignore presque complètement la façon dont les rapports entre la fabrication des cloches et la conception des clochers furent calculés préalablement par les maîtres d'œuvre mais un tel déficit est également vrai pour la conception d'autres parties d'un édifice médiéval. Il faut donc

I3 Claus Peter, «Die Glocken der Bamberger Altstadt. Teil I.I : Das Domgeläute», Jabrbuch der Glockenkunde, 9-10, 1997-1998, p. 13-29. 
se concentrer sur l'analyse archéologique, tout en se rendant compte de problèmes délicats en matière de conservation : cloches et breffois ont souvent été modifiés, détruits et renouvelés; de même, la documentation concernant l'accompagnement et la structuration du déroulement liturgique et cérémoniel par les cloches est souvent très fragmentaire. Il s'agit donc ici d'étudier à titre d'exemples quelques cas remarquables tout en restant prudent sur la question de leur généralisation.

L'ancienne collégiale Saint-Barthélemy à Francfort a bénéficié de profondes recherches campanologiques, dues à Konrad Bund, un des meilleures spécialistes dans ce domain $\mathrm{e}^{14}$. La construction du nouveau clocher gothique, commencée en I4I5, fut conçue comme une seule tour très haute, érigée à l'ouest de la nef beaucoup plus ancienne, en remplacement d'un massif occidental du XII ${ }^{\mathrm{e}}$ siècle (fig. I). Juste avant le début des travaux, les vieilles cloches furent transférées dans un beffroi provisoire, érigée dans l'enclos canonial, près de la nouvelle construction.

Au fur et à mesure de l'avancement des travaux à la tour, les cloches étaient suspendues à des beffrois provisoirement installés dans les nouveaux étages. À partir de 1440 environ, lorsque le deuxième étage de la tour était en cours de construction, on commença la fonte de nouvelles cloches. En l'espace d'une décennie, on refit donc non seulement la tour mais aussi la sonnerie qui atteignit finalement le nombre de neuf cloches. Tout ceci fut apparemment conçu avant le début de la construction car les voûtains du rez-de-chaussée de la tour montrent une ouverture d'un diamètre qui correspond à celui de la plus grande cloche. Au moment du voûtement du dernier étage, en 1507 , toutes les cloches obtenaient finalement leur place définitive dans la chambre octogonale, très haute, de la tour. Le beffroi était composée de deux parties superposées abritant en bas les cloches de la collégiale et au-dessus celles du conseil communal («Rat ») (fig. 2). En somme, cette charpente double remplissait complètement l'étage octogonal de la tour, la partie la plus remarquable de tout l'édifice. Sa fonction en tant que chambre des cloches est encore renforcée par les grandes ouvertures qui s'élèvent sur toute la hauteur de l'octogone. Bien évidemment, elles ont pour fonction de laisser s'échapper les sons et de les diriger mais leur importance est également rendue visible par la couronne de gables qui règne au-dessus des lancettes. Etant donné toutes ces corrélations étroites entre le nombre des cloches, l'espace qu'elles occupent et la structure architecturale du clocher de Francfort, force est de conclure qu'il s'agit d'une programmation concertée qui englobait à la fois la conception de la sonnerie et de l'architecture. Autrement dit, l'architecture, dans ses données techniques et sa composition esthétique, répond dans une large mesure à sa fonction de «phare acoustique ». Plus concrètement, la mise en valeur de l'étage de

I4 Konrad Bund, Frankfurter Glockenbuch, Francfort-sur-le-Main, Kramer, 1986 (« Mitteilungen aus dem Frankfurter Stadtarchiv », 4). 


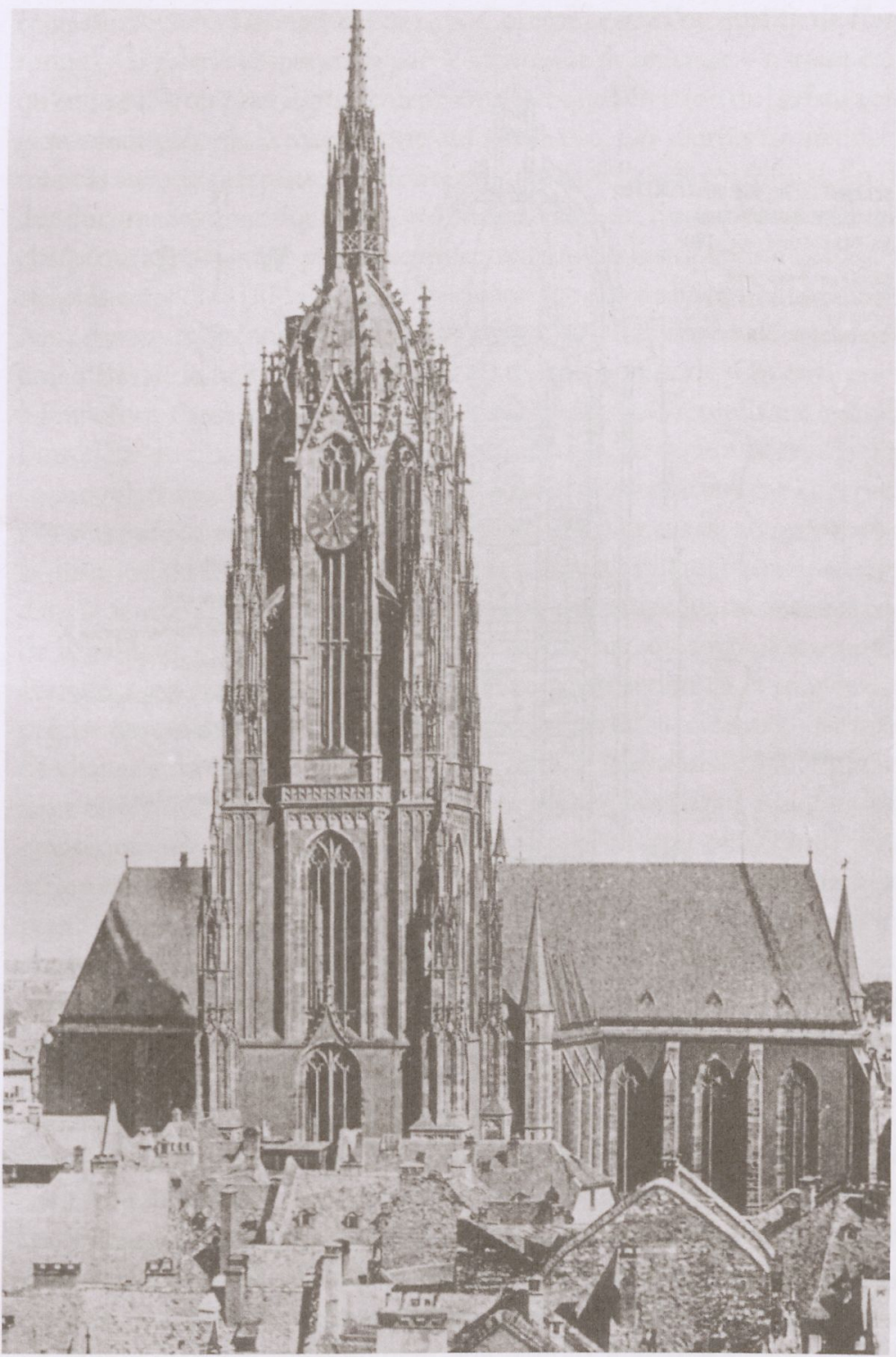

Fig. I. Francfort, ancienne église collégiale et paroissiale Saint-Barthélemy, clocher, état vers 1900 (cl. Chr. Freigang).

l'octogone, qui contraste avec le soubassement carré par son plan octogonal et son ornementation plus riche, est destinée à signaler visuellement l'endroit d'où parlent les voix sacrées des cloches. 


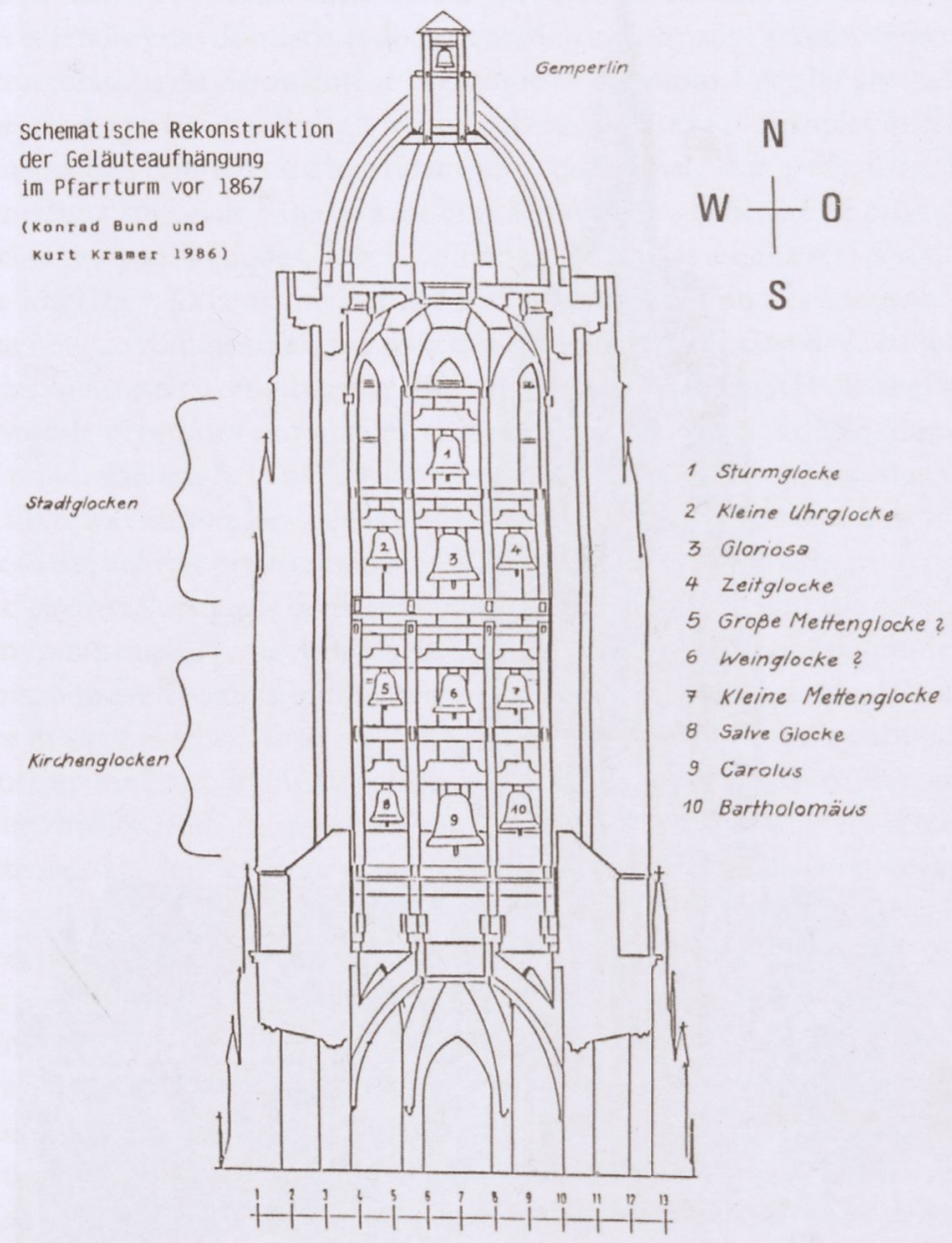

Fig. 2. Francfort-sur-le-Main, ancienne collégiale Saint-Barthélemy, coupe de l'octogone avec le beffroi en charpente, état avant 1867 (d'après K. BUND éd., Frankfurter Glockenbuch, Francfort-sur-le-Main, Verlag Waldemar Kramer, 1986, p. 252).

Le clocher de la très ambitieuse église paroissiale d'Ulm (le soidisant Münster) permet d'approfondir ces observations. Comme à Francfort, la construction de la tour, commencée en 1391, allait de pair avec la fabrication de trois nouvelles cloches tout aussi ambitieuses, au milieu du XV siècle, afin de compléter la sonnerie déjà existante de deux instruments fondus au siècle précédent (planche 59). Finalement, la sonnerie médiévale comptait six cloches, suspendues au troisième 
étage du clocher et inaugurées en I455. Comme ce que l'on qualifie de Kranz (couronne) - la galerie entourant la partie supérieure de cet étage - n'ayant été achevée qu'en $1494-$, on peut légitimement supposer que l'érection du beffroi a chronologiquement précédé la maçonnerie qui l'enchâsse. Les sources ne mentionnent en tout cas aucune charpente provisoire montée dans l'enclos paroissial. En revanche, des documents graphiques de 1596 insérés dans un devis pour la réfection de la charpente apportent de précieuses informations sur la suspension des cloches avant et après cette date (fig. 3$)^{15}$. Une charpente de $14 \mathrm{~m}$ de hauteur était calée à l'intérieur des murs latéraux du troisième étage. Sur cette œuvre de soubassement en bois s'élevait le beffroi proprement dit, d'une hauteur de $6 \mathrm{~m}$ environ. Comme à Francfort, l'ensemble de cette construction en bois remplissait complètement l'intérieur du troisième étage du clocher, entre le Steinerner Boden (la plateforme en pierre), servant de sol de l'étage et le Kranz. C'est exactement à cet endroit que s'ouvrent d'imposantes double-arcades, sur une hauteur de $20 \mathrm{~m}$, pour permettre la diffusion du son de cloches. On arrive même à expliquer certaines répartitions dans le fenestrage, notamment une traverse qui coupe les meneaux au deux-tiers de la hauteur, car c'est là que le beffroi repose sur son soubassement. Comme à Francfort, on prévoyait dès le début de la construction de la tour la dimension précise des cloches car la voûte du porche du portail occidental - formant le rezde-chaussée du clocher - ainsi que le Steinerner Boden montrent chacun une très large ouverture ronde d'un diamètre de 7 pieds, équivalent à $2 \mathrm{~m}$. Les documents graphiques nous renseignement également sur l'endroit où l'équipe de sonneurs actionnaient les cordes: ils les tiraient au rez-de-chaussée, directement derrière le grand portail occidental, et leur action se faisait donc publiquement, voire à un endroit privilégié. Cela nous permet de conclure qu'avec la construction du troisième étage de la tour, tous ses composants fonctionnels étaient achevés d'après une conception élaborée dès le début: le portail avec son programme iconographique puis le premier étage avec sa très grande baie occidentale dédiée à Saint-Martin et sa tribune donnant sur la nef, et finalement, dans la zone supérieure, l'étage très élevé entièrement consacré à loger les cloches, le pourtour du Kranz et une maisonnette pour les gardiens de la tour, aménagée sur la plateforme supérieure de cet étage.

Toutes les données architecturales du troisième étage correspondent incontestablement à sa fonction essentielle d'abri pour ses immenses instruments de musique. Le plan de la tour reste, jusqu’à cet étage, rectangulaire pour créer un espace intérieur suffisamment ample pour le beffroi et son soubassement. À l'étage supérieur, conçu sur une série d'épures au Moyen Âge mais pas réalisé avant le XIX' siècle, le plan se transforme en octogone et l'espace disponible se réduit très sensiblement. De même, les contreforts de la tour, très saillants jusqu'à

Is Stadtarchiv Ulm A [1618]. 


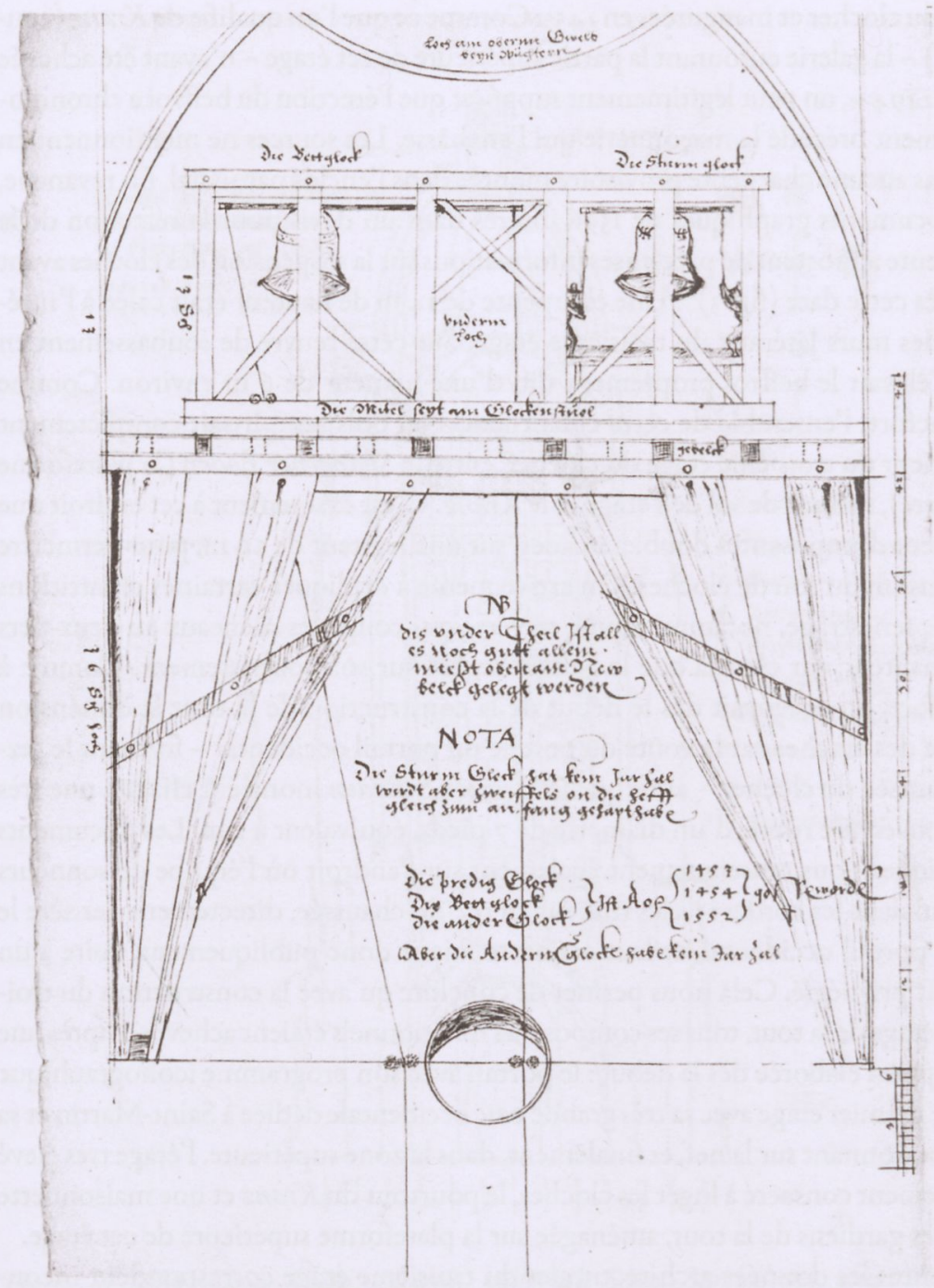

Fig. 3. Ulm, église paroissiale «Münster», projet de réfection du beffroi en charpente (Is96; cl. Ulm, Stadtarchiv A [I6I8].)

l'étage des cloches, se rétrécissent dans les niveaux supérieurs. De plus, toutes les épures montrent, au-dessus de la chambre des cloches, une nette rupture de conception. À partir de ce niveau, on prévoit un octogone d'une légèreté époustouflante, composé par des remplages en filigrane et nettement en retrait par rapport au massif situé au-dessous. Le couronnement du clocher d'une hauteur de quelques 80 mètres par l'étage de l'octogone et une flèche aussi audacieuse n'a 
pas de fonction pratique mais elle est destinée à être vue de très loin et, surtout, à couronner les cloches par une sorte de baldaquin monumental. Le clocher d'Ulm ressemble donc à un ostensoir ou à un des tabernacles du gothique tardif, avec ses immenses flèches au-dessus du réceptacle du sacrement. À l'endroit normalement réservé au sacrement, on trouve, dans le clocher, les instruments des voix sacrées.

Un troisième exemple, plus ancien mais bien documenté et conservé, nous est donné par les cloches et le clocher de la paroissiale (Münster) de Fribourg-enBrisgau. La grande cloche « Hosanna », qui existe toujours, fut fondue en 1258 , une deuxième suivit en I28I et le très haut beffroi fut érigé en I291, au-dessus de l'étage de l'horloge (fig. 4, planche 60). L'étude archéologique montre que l'œuvre de charpenterie fut montée avant les murs. Comme dans d'autres cas, par exemple à la cathédrale d'Utrecht, le beffroi s'élevait d'abord en plein air, pleinement visible pendant un certain laps de temps de quelques années ou décennies. Si l'étage de l'octogone qui abrite les cloches a été depuis toujours loué en raison de sa forme en filigrane rappelant celle d'un ciboire en métal, sa conception répond, encore une fois, à la mise en place des cloches. « Hosanna » se trouve dans la moitié supérieure de l'octogone, marquée à l'extérieur par une corniche traversant les ouvertures. Leurs parties basses sont murées mais au-dessus le son des cloches peut se répandre largement et ces ouvertures d'échappement sont même encadrées par d'élégants gâbles. Au-dessus, l'étage est couvert par une voûte surbaissée, sorte d'abat-son destiné à diriger les sons vers la ville. La partie supérieure de l'octogone s'ouvre en filigrane, comme un tabernacle, et elle est surmontée par la fameuse flèche à remplage. Toutes les parties s'avèrent, dans leur conception constructive, étroitement liées entre elles. Le beffroi commence dès l'étage de l'horloge, à un niveau où la maçonnerie est encore très massive et où elle circonscrit un plan carré. En hauteur, la charpente se dresse, en se rétrécissant, dans l'intérieur de l'octogone beaucoup plus ajouré et décoré pour laisser échapper et pour visualiser les sons des cloches. À Fribourg, cette fonction est clairement soulignée par un cycle de grandes statues qui orne les faces latérales des contreforts « en éperon », exactement au niveau des cloches (planche 60). Il s'agit de prophètes, chacun encadré par une large arcade qui correspond aux ouvertures du son à côté. Ce voisinage entre cloches et prophètes est certainement issu de l'interprétation exégétique, mentionnée plus haut, du son des cloches comme voix puissante des prophètes. Le clocher de Fribourg confirme donc encore une fois l'étroir rapport rapprochant la conception technique, la forme architecturale et l'emplacement ainsi que la mise en scène des cloches au Moyen Âge. Peut-être pourrait-on aller jusqu'à proposer une explication pour les flèches largement ajourées non seulement à Ulm et à Fribourg mais aussi à Cologne, Meissen, Strasbourg, Berne, etc. Leur structure largement ouverte révèle non seulement une évidente virtuosité technique mais aussi une perméabilité symbolique des murs destinée à laisser le son sacré des cloches s'échapper librement vers le ciel. 


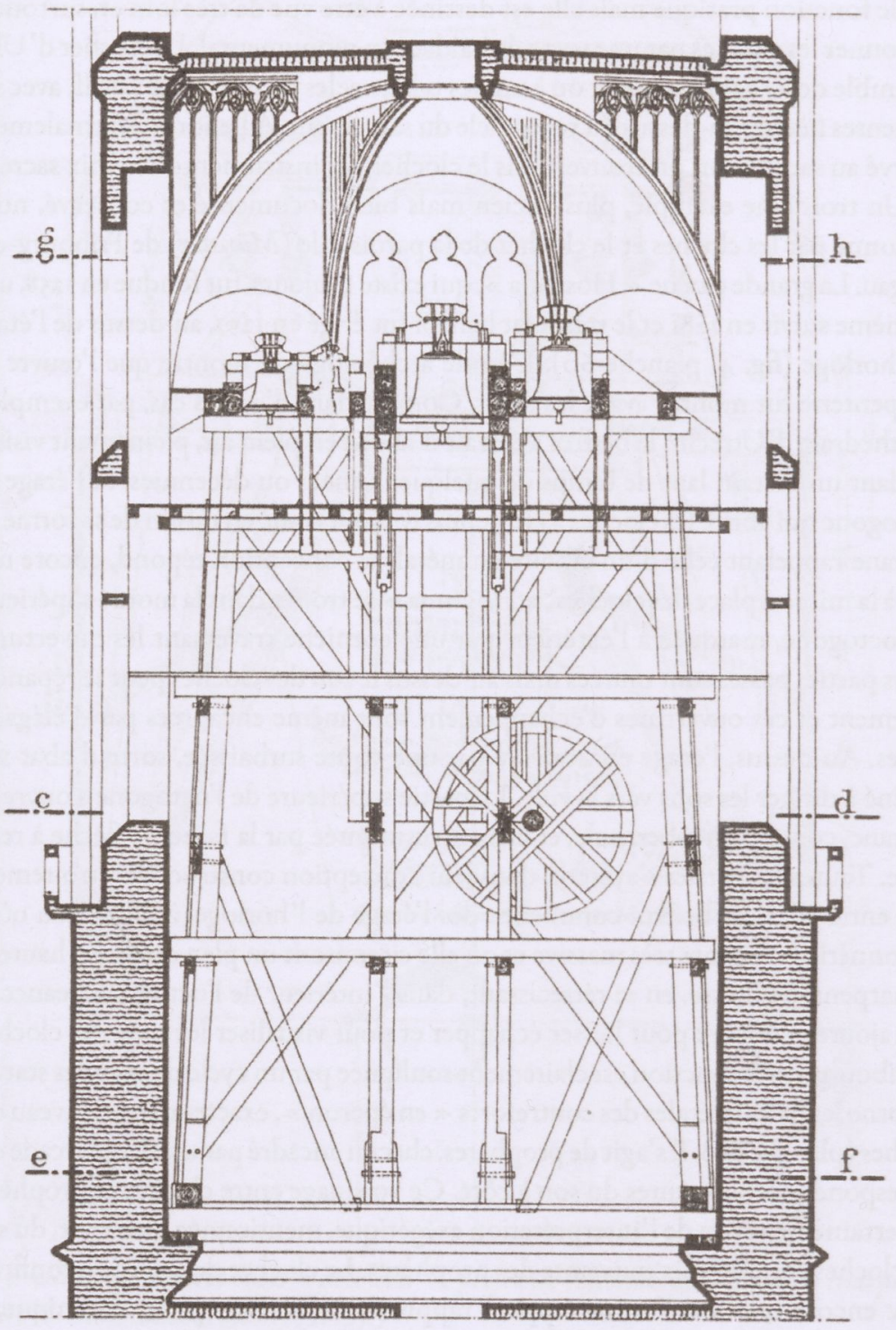

Fig. 4. Fribourg-en-Brisgau, église paroissiale, «Münster», charpente des cloches dans le clocher (d'après F. ADLER, «Das Münster zu Freiburg i. Br. », dans Deutsche Bauzeitung, IS/I88I, p. 505). 


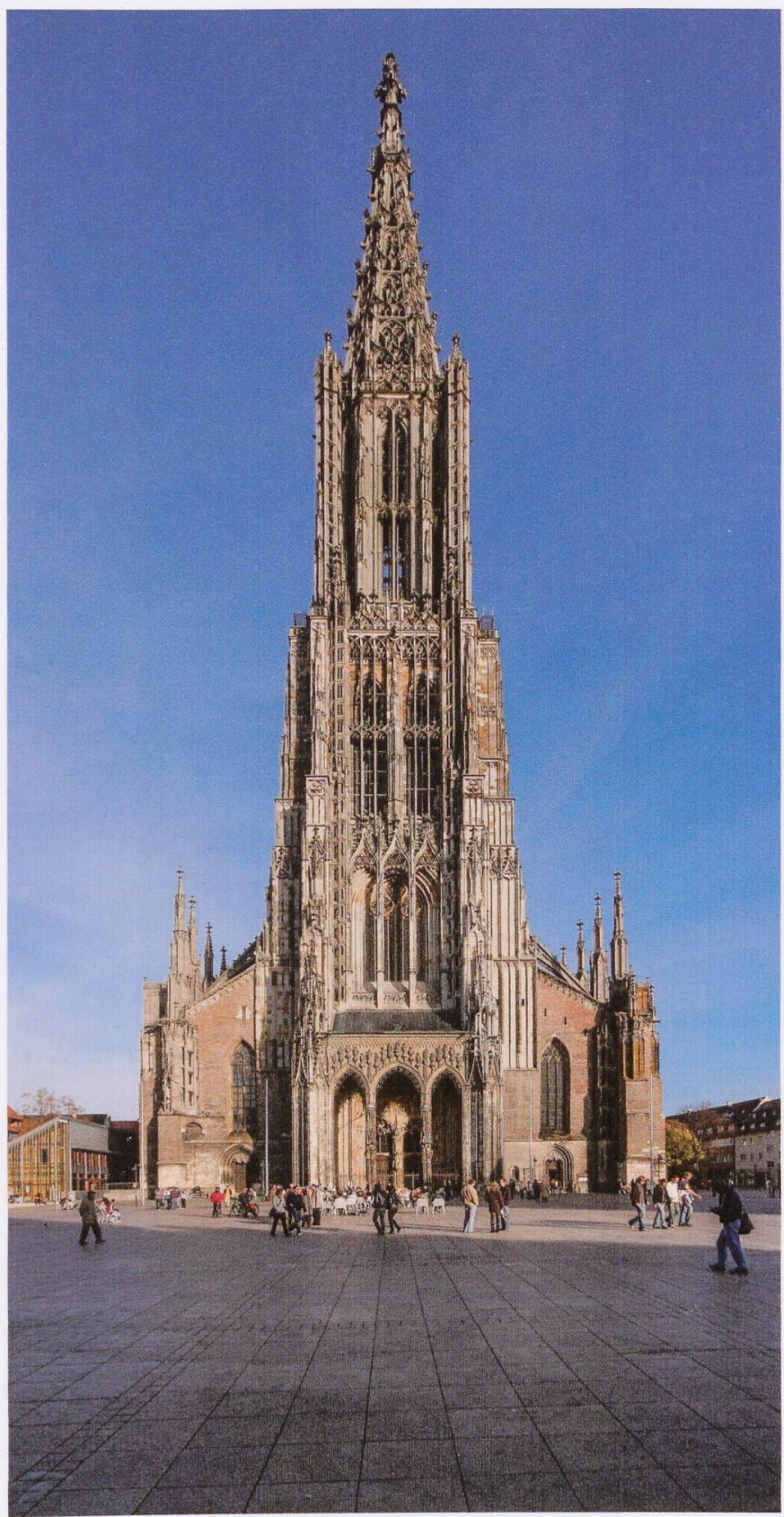

Pl. 59 


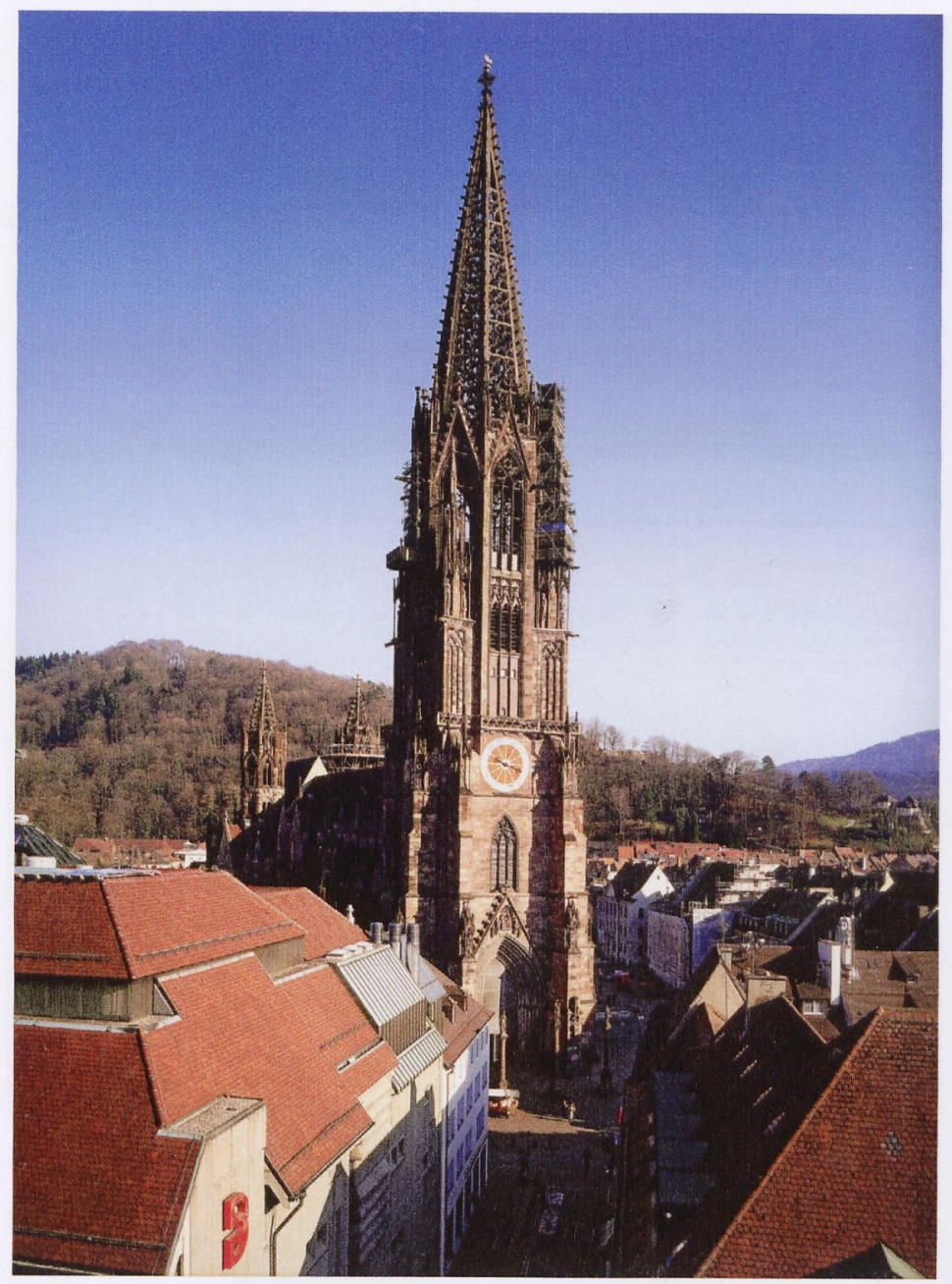

Pl. 60 